\title{
Changed pattern of hospital admission in stroke during COVID-19 pandemic period in Iran: a retrospective study
}

\author{
Roozbeh Tavanaei ${ }^{1} \cdot$ Kaveh Oraii Yazdani $^{2} \cdot$ Mohammadhosein Akhlaghpasand $^{1} \cdot$ Alireza Zali $^{1}$. \\ Saeed Oraee-Yazdani ${ }^{1}$ (D)
}

Received: 22 September 2020 / Accepted: 24 December 2020 / Published online: 4 January 2021

(C) Fondazione Società Italiana di Neurologia 2021

\begin{abstract}
Background Some previous reports have shown a reduced number of admission in stroke cases during the coronavirus disease 2019 (COVID-19) pandemic period. The present study aimed to investigate this changing pattern and the potential causes behind it at an academic neurology and neurosurgery center in Iran.

Methods Patients admitted to our center with the diagnosis of ischemic and hemorrhagic stroke, between March 1, 2019, Jun 1, 2019, and the similar 3-month period in 2020 (COVID-19 pandemic period), were compared in terms of clinical characteristics and outcome. Poisson regression was also conducted to assess the correlation between daily admissions and the COVID-19 pandemic period.

Results A total of 210 patients with stroke (ischemic and hemorrhagic) in 2019 were compared with 106 patients in 2020. COVID-19 pandemic period was significantly associated with the decline in the number of daily admissions in ischemic stroke (IRR, 0.51 [95\% CI, 0.4-0.64]). A significant reduction $(P=0.003)$ in time from onset to arrival at hospital from median $12 \mathrm{~h}$ [IQR, 5-32] in 2019 to median $6 \mathrm{~h}$ [IQR, 4-16] in 2020 was found in ischemic stroke cases. National Institute of Health Stroke Scale (NIHSS) was significantly increased $(P<0.001)$ from median 4 [IQR, 2-7] in 2019 to median 9 [IQR, 4-14] in 2020. Glasgow coma scale (GCS) was significantly decreased from 13.9 (SD, 2) in 2019 to 12.8 (SD, 2.9) in $2020(P<0.001)$.

Conclusions The present study provided new pieces of evidence regarding the changed pattern of hospital admission in stroke especially the possible reasons for its decline.
\end{abstract}

Keywords Coronavirus $\cdot$ Stroke $\cdot$ Pandemics $\cdot$ Cerebrovascular disease $\cdot$ Public health $\cdot$ Subarachnoid hemorrhage Hospitalization

\section{Introduction}

The ongoing COVID-19 pandemic has changed the face of healthcare systems globally. Since the start of the pandemic, many countries have implemented lockdown and physical distancing measures in order to reduce the spread of COVID-19. However, this strategy as well as the fear of

Saeed Oraee-Yazdani

Saeed_o_yazdani@sbmu.ac.ir

1 Functional Neurosurgery Research Center, Shohada Tajrish Comprehensive Neurosurgical Center of Excellence, Shahid Beheshti University of Medical Sciences, Tehran, Iran

2 Department of Cardiovascular Diseases, Zahedan University of Medical Science, Zahedan, Iran
COVID-19 infection has resulted in patients' hesitation to seek medical emergency care. Previous reports have shown a significant drop in the number of admissions in patients presenting with acute coronary syndromes and ischemic stroke during the COVID-19 pandemic in comparison to the previous year [1-4]. This could finally result in increased outof-hospital morbidity, mortality, and a poorer prognosis upon presentation. Moreover, additional workload imposed on emergency facilities by COVID-19 cases mandates the need for major reorganizations in the healthcare system to decrease the complications associated with other urgent conditions.

Worldwide, stroke is one of the leading causes of mortality and morbidity. Acute stroke interventions and outcomes of the patients are highly dependent on the time from symptom onset to arrival which can be prolonged during the pandemic [5-7]. The objective of the present study was to examine the changes in the pattern of hospital admissions for ischemic and 
hemorrhagic stroke during the COVID-19 pandemic at a neurology and neurosurgery center in Iran.

\section{Methods}

This retrospective cohort study was performed at Shohada Tajrish Hospital and approved by the institutional review board of Shahid Beheshti University of Medical Sciences. Our center is one of the three academic public comprehensive stroke unit hospitals providing acute stroke care for northern, north-eastern, and eastern regions of Tehran province and city (covering more than 5.7 million inhabitants with approximately $80 \%$ in Tehran city). Our stroke network follows a centralized management system that is suitable for sectors with a high population density in metropolitan areas. All the eligible patients in our territory are transferred by the ambulance services to these three hospitals 24/7, following the emergency medical services (EMS) teleconsultation. Informed consent was not required due to the retrospective nature of the study. The COVID-19 outbreak in Iran was officially announced on February 19, 2020, following the death of two patients in Qom city, due to COVID-19 infection. Thus, patients with the diagnosis of ischemic stroke, transient ischemic attack (TIA), and hemorrhagic stroke including non-traumatic subarachnoid hemorrhage (SAH) and intracerebral hemorrhage (ICH), in 3 months between March 1, 2020, Jun 1, 2020, and the corresponding population to this group in the previous year between March 1, 2019, and Jun 1, 2019, were included in this study. Patients' data were gathered from the medical records of the Shohada Tajrish Hospital. Data extraction from medical records was performed according to ICD 10 discharge codes which included ischemic or hemorrhagic strokes (160.x, 161.x, and 163.x) and transient ischemic attack (TIA, G45.x).

Patients in the two groups (periods) were comparatively analyzed in terms of demographics (age, sex), time from onset to arrival at the hospital, type of stroke (ischemic/TIA and hemorrhagic), treatment, comorbidities, in-hospital mortality, GCS, NIHSS, and onset symptoms. Stroke severity was classified based on NIHSS as mild (0-4), moderate (5-8), and severe ( $>8)$. NIHSS and GCS scores are according to patients' clinical status upon presentation. Furthermore, these variables were also compared between the ischemic stroke patients with and without COVID-19 infection. COVID-19 diagnosis was performed using quantitative reverse-transcriptase polymerase chain reaction (RT-PCR) test of nasopharyngeal swab samples.

\section{Statistical analysis}

Quantitative variables (age, time from onset to arrival, GCS, and NIHSS) were presented as mean and standard deviation
(SD) or median and inter-quartile range (IQR, 25th to 75th percentile). Nominal variables were expressed as frequency and percentage. Mann-Whitney $U$ test was performed to compare differences in continuous variables between the two groups. Chi-square and Fisher's exact tests were used to assess differences in categorical variables. Furthermore, in order to determine whether there was a significant difference in the daily admissions between the two study periods, incidence rate ratios (IRRs) with 95\% confidence interval (CI) were calculated using Poisson regression (daily admissions as the response variable; year, month, and day as the independent variables). Statistical analysis was performed using SPSS 24 (SPSS, Inc., Chicago, IL, USA). Poisson regression was performed using STATA 15. $P$ values $<0.05$ were considered statistically significant.

\section{Results}

From March 1, 2019, to Jun 1, 2019, a total of 210 cases of stroke (ischemic and hemorrhagic) were admitted to our center, and a total of 106 cases were diagnosed during the same period in 2020 (COVID-19 pandemic). There is a reduction of $50 \%$ in all types of stroke in our center in comparison to 2019 (Figs. 1 and 2). Details regarding demographics and clinical characteristics of patients with ischemic and hemorrhagic stroke are depicted in Tables 1 and 2, respectively.

Time from onset to arrival among cases with ischemic stroke was significantly reduced in 2020 (median, $6 \mathrm{~h}$ [IQR, 4-16], $P=0.003$ ) compared with 2019 (median, $12 \mathrm{~h}$ [IQR, 5-32]). There was no significant difference in time from onset to arrival in hemorrhagic cases $(P=0.64)$. A significant decrease $(P<0.001)$ in GCS scores in 2020 with an average of $12.8(\mathrm{SD}, 2.9)$ in comparison to 2019 with an average of 13.9 (SD, 2) for all cases was found. Percentage of cases with GCS score $13-15$ was decreased from 80.9 to $64.8 \%$, GCS score 9 12 was increased from 16.4 to $26.4 \%$, GCS score 6-8 was increased from 2.2 to $4.4 \%$, and GCS score $3-5$ was increased from 0.5 to $4.4 \%$. GCS scores were similar for hemorrhagic stroke in both periods $(P=0.6)$. There was a significant increase in NIHSS scores in 2020 (median, 9 [IQR, 4-14], $P<$ 0.001) compared with 2019 (median, 4 [IQR, 2-7]) for the ischemic stroke. NIHSS score $0-4$ was decreased from 51.9 to $21.8 \%$, NIHSS score 5-8 was decreased from 29.6 to $23.7 \%$, NIHSS score 9-14 was increased from 11.1 to $29 \%$, and NIHSS score $>14$ was increased from 7.4 to $21.5 \%$ in COVID-19 pandemic period.

No statistically significant change was observed in stroke symptoms from 2019 to 2020, except for a significant increase in motor symptoms (an increase of $16.3 \%, P=0.003$ ) and impaired level of consciousness (an increase of $20 \%, P<$ $0.001)$ during the pandemic period. There was a significant increase $(11.7 \%, P=0.007)$ in the mortality rate for all cases 
Table 1 Clinical and demographic characteristics of patients with ischemic stroke in pre-COVID-19 and COVID-19 period

\begin{tabular}{|c|c|c|c|}
\hline & 2019 (Mar 1 to Jun 1$)$ & 2020 (Mar 1 to Jun 1$)$ & $P$ value \\
\hline Number of admissions & 190 & 95 & \\
\hline COVID-19 infection $(n, \%)$ & - & $15(15.8 \%)$ & \\
\hline Age (mean, $\mathrm{SD}^{1}$ [years]) & $67.1(14.9)$ & $68.3(15.2)$ & 0.44 \\
\hline Sex $($ male $[n, \%])$ & $111(58.4 \%)$ & $50(52.6 \%)$ & 0.60 \\
\hline Time from onset to arrival at hospital (median $[\mathrm{IQR}]^{2}$, hours) & $12(5-32)$ & $6(4-16)$ & $0.003 *$ \\
\hline Time from arrival to thrombolysis (median [IQR], minutes) & $18(15-31)$ & $22(20-45)$ & 0.42 \\
\hline Time from arrival to puncture (median [IQR], minutes) & $123(107-135)$ & $127(105-140)$ & 0.66 \\
\hline \multicolumn{4}{|l|}{ Comorbidities $(n, \%)$} \\
\hline Hypertension & $84(44.2 \%)$ & $53(55.8 \%)$ & $0.07 *$ \\
\hline Diabetes mellitus & $65(34.2 \%)$ & $30(31.6 \%)$ & 0.65 \\
\hline Coronary artery disease & $35(18.4 \%)$ & $26(27.4 \%)$ & $0.08 *$ \\
\hline Heart failure & $23(12.1 \%)$ & $8(8.4 \%)$ & 0.34 \\
\hline Atrial fibrillation & $27(14.2 \%)$ & $18(18.9 \%)$ & 0.30 \\
\hline Renal disease & $6(3.2 \%)$ & $2(2.1 \%)$ & 0.61 \\
\hline Prior stroke & $33(17.4 \%)$ & $14(14.7 \%)$ & 0.57 \\
\hline Cancer & $2(1.1 \%)$ & $1(1.1 \%)$ & 1.00 \\
\hline Arterial territory (anterior/middle $[n, \%]$ ) & $124(65.3 \%)$ & $67(70.5 \%)$ & $0.04 *$ \\
\hline \multicolumn{4}{|l|}{ Stroke subtypes $(\mathrm{n}, \%)$} \\
\hline Large artery atherosclerosis & $24(12.6 \%)$ & $16(16.8 \%)$ & 0.33 \\
\hline Cardioembolic & $47(24.7 \%)$ & $22(23.2 \%)$ & 0.76 \\
\hline Small vessel disease & $71(37.4 \%)$ & $36(37.9 \%)$ & 0.93 \\
\hline Other determined etiology & $5(2.6 \%)$ & $0(0.0 \%)$ & 0.17 \\
\hline Cryptogenic or undetermined & $43(22.6 \%)$ & $21(22.1 \%)$ & 0.92 \\
\hline \multicolumn{4}{|l|}{ Symptoms (n, \%) } \\
\hline Impaired level of consciousness & $52(27.4 \%)$ & $45(47.4 \%)$ & $0.001^{*}$ \\
\hline Motor symptoms & $115(60.5 \%)$ & $73(76.8 \%)$ & $0.006^{*}$ \\
\hline Sensory deficit & $4(2.1 \%)$ & $3(3.2 \%)$ & 0.58 \\
\hline Visual field loss & $11(5.8 \%)$ & $6(6.3 \%)$ & 0.86 \\
\hline Aphasia & $42(22.1 \%)$ & $23(24.2 \%)$ & 0.70 \\
\hline Dysarthria & $85(44.7 \%)$ & $38(40.0 \%)$ & 0.44 \\
\hline Ataxia & $27(14.2 \%)$ & $7(7.4 \%)$ & 0.09 \\
\hline \multicolumn{4}{|l|}{ Treatment interventions $(n, \%)$} \\
\hline Thrombolysis & $25(13.2 \%)$ & $18(18.9 \%)$ & 0.19 \\
\hline Thrombectomy & $6(3.2 \%)$ & $7(7.4 \%)$ & 0.10 \\
\hline Thrombolysis and thrombectomy & $10(5.3 \%)$ & $7(7.4 \%)$ & 0.47 \\
\hline \multicolumn{4}{|l|}{$\operatorname{NIHSS}^{3}(\mathrm{n}, \%)$} \\
\hline Median (IQR) & $4(2-7)$ & $9(4-14)$ & $<0.001 *$ \\
\hline $0-4$ & $98(51.6 \%)$ & $24(25.3 \%)$ & $<0.001^{*}$ \\
\hline $5-8$ & $56(29.5 \%)$ & $22(23.2 \%)$ & 0.29 \\
\hline $9-14$ & $21(11.1 \%)$ & $27(28.4 \%)$ & $<0.001 *$ \\
\hline$>14$ & $14(7.4 \%)$ & $20(21.1 \%)$ & $0.001^{*}$ \\
\hline \multicolumn{4}{|l|}{$\operatorname{GCS}^{4}(n, \%)$} \\
\hline Mean (SD) & $13.85(2.01)$ & $12.78(2.79)$ & $<0.001^{*}$ \\
\hline $3-5$ & $1(0.5 \%)$ & $4(4.4 \%)$ & 0.52 \\
\hline $6-8$ & $4(2.2 \%)$ & $4(4.4 \%)$ & 0.31 \\
\hline $9-12$ & $31(16.4 \%)$ & $25(26.4 \%)$ & $0.04 *$ \\
\hline $13-15$ & $154(80.9 \%)$ & $62(64.8 \%)$ & $0.003^{*}$ \\
\hline Decompressive craniectomy $(n, \%)$ & $2(1.1 \% \%)$ & $2(2.1 \%)$ & 0.47 \\
\hline In-hospital mortality $(n, \%)$ & $14(7.4 \%)$ & $16(16.8 \%)$ & $0.01 *$ \\
\hline
\end{tabular}

*indicates statistically significant $(P<0.05)$

${ }^{1}$ Standard deviation

${ }^{2}$ Interquartile range

${ }^{3}$ National Institute of Health Stroke Scale

${ }^{4}$ Glasgow coma scale

(both ischemic and hemorrhagic) in 2020. However, no significant difference in the mortality rate for hemorrhagic stroke was found $(P=0.06)$.

There was no significant change in ischemic stroke treatments including thrombolysis $(P=0.19)$ and thrombectomy
$(P=0.10)$. The frequency of decompressive craniectomy was similar between the two periods $(P=0.47)$. No significant change in the time from arrival to thrombolysis $(P=0.42)$ and puncture $(P=0.66)$ was observed in the COVID-19 pandemic period compared with the pre-COVID-19 period. 
Poisson regression showed a correlation between the reduction in ischemic stroke admissions and the COVID-19 pandemic period (IRR of year, 0.51 [95\% CI, 0.4-0.64]). IRRs for month and day were 1.17 [95\%CI, 1-1.24] and 0.98 [95\%CI, 0.97-0.99]. However, no significant association between hemorrhagic stroke (non-traumatic SAH and ICH) admissions and COVID-19 period was found (IRR of year, 0.55 [95\% CI, 0.26-1.14]). Moreover, Poisson regression was used for the association between the COVID-19 pandemic period and daily admissions of severe stroke cases (NIHSS scores $>9$ ) which showed no significant correlation (IRR, 1,34 [95\% CI, 0.86-2.04]). Table 3 shows the IRRs calculated by Poisson regression.

Fifteen cases of ischemic stroke and 3 cases of SAH are diagnosed with COVID-19 infection (onset before the hospital admission or hospitalization) whose demographic and clinical characteristics are shown in Table 4. Patients with both COVID-19 infection and ischemic stroke had a significantly higher NIHSS score (median, 14 [IQR, 9.5-16.5]) in comparison to non-infected ones (median, 7.5 [IQR, 4-13], $P=0.01$ ). No significant difference in the number of NIHSS subgroups was found between the infected and non-infected cases. GCS scores were significantly lower $(P<0.001)$ in infected patients 10.27 (SD, 2.74) compared with non-infected ones 13.18 (SD, 2.60). The in-hospital mortality rate was also significantly higher in infected patients $(P=0.04)$. Among the 15 patients with COVID-19 infection, 11 had symptoms with a median time from COVID-19 symptoms to the onset of stroke of 6.5 [IQR, 2-15]. Regarding the stroke subtypes, small vessel disease and cryptogenic categories were significantly lower $(P=$ $0.001)$ and higher $(P=0.004)$ in stroke cases with COVID-19 infection compared with non-infected patients, respectively.

\section{Discussion}

The current study showed that there is a significant association between the COVID-19 pandemic period and a reduction in the number of admissions in ischemic stroke compared with the same period of 2019. A significant increase in the severe presentations (NIHSS scores $\geq 9$ ) and a significant reduction in the mild to moderate presentations (NIHSS scores $0-8$ ) of
Table 2 Clinical and demographic characteristics of patients with hemorrhagic stroke in pre-COVID-19 and COVID-19 period

\begin{tabular}{|c|c|c|c|}
\hline & 2019 (Mar 1 to Jun 1) & 2020 (Mar 1 to Jun 1$)$ & $P$ value \\
\hline Number of admissions & 20 & 11 & \\
\hline COVID-19 infection $(n, \%)$ & - & $3(27.3 \%)$ & \\
\hline Age (mean, $\mathrm{SD}^{1}$ [years]) & $55.9(14.2)$ & $51.1(12.4)$ & 0.67 \\
\hline Sex $($ male $[n, \%])$ & $14(65.0 \%)$ & $10(90.9 \%)$ & $0.04 *$ \\
\hline $\begin{array}{l}\text { Time from onset to arrival at } \\
\text { hospital (median }[\mathrm{IQR}]^{2} \text {, hours) }\end{array}$ & $10(4-72)$ & $15(10-72)$ & 0.64 \\
\hline \multicolumn{4}{|l|}{ Comorbidities $(n, \%)$} \\
\hline Hypertension & $7(35.0 \%)$ & $3(27.3 \%)$ & 0.40 \\
\hline Diabetes mellitus & $1(5.0 \%)$ & $1(9.1 \%)$ & 0.65 \\
\hline Coronary artery disease & $1(5.0 \%)$ & $0(0.0 \%)$ & 0.17 \\
\hline Prior stroke & $1(5.0 \%)$ & $3(27.3 \%)$ & 0.63 \\
\hline \multicolumn{4}{|l|}{ Stroke type $(n, \%)$} \\
\hline $\mathrm{aSAH}^{3}$ & $16(80.0 \%)$ & $9(81.8 \%)$ & 0.90 \\
\hline $\mathrm{ICH}^{4}$ & $4(20.0 \%)$ & $2(18.2 \%)$ & 0.90 \\
\hline \multicolumn{4}{|l|}{$\operatorname{GCS}^{5}(n, \%)$} \\
\hline Mean (SD) & $11.71(4.24)$ & $12.85(3.65)$ & 0.06 \\
\hline $3-5$ & $2(10.0 \%)$ & $4(36.4 \%)$ & $0.07 *$ \\
\hline $6-8$ & $1(5.0 \%)$ & $0(0.0 \%)$ & 0.45 \\
\hline $9-12$ & $2(10.0 \%)$ & $3(27.3 \%)$ & 0.21 \\
\hline $13-15$ & $15(75.0 \%)$ & $4(36.4 \%)$ & $0.03 *$ \\
\hline In-hospital mortality $(n, \%)$ & $3(15.0 \%)$ & $5(45.4 \%)$ & 0.06 \\
\hline \multicolumn{4}{|c|}{ *indicates statistically significant $(P<0.05)$} \\
\hline \multicolumn{4}{|l|}{${ }^{1}$ Standard deviation } \\
\hline \multicolumn{4}{|l|}{${ }^{2}$ Interquartile range } \\
\hline \multicolumn{4}{|l|}{${ }^{3}$ Aneurysmal subarachnoid hemorrhage } \\
\hline \multicolumn{4}{|l|}{${ }^{4}$ Intracerebral hemorrhage } \\
\hline${ }^{5}$ Glasgow coma scale & & & \\
\hline
\end{tabular}


Fig. 1 Stroke admissions according to their type during the same periods in 2019 and 2020 (COVID-19 pandemic period). IS, ischemic stroke; SAH, subarachnoid hemorrhage; ICH, intracerebral hemorrhage.

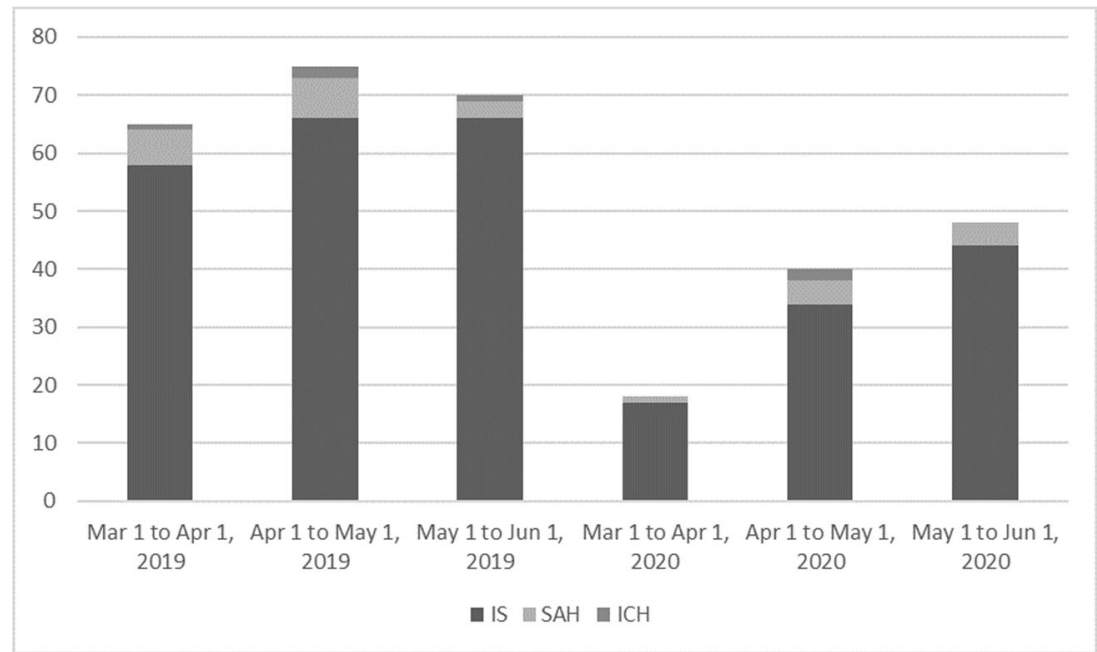

ischemic stroke were found. The significantly decreased time from onset to arrival, during the COVID-19 pandemic, was also one of our findings.

Similar to our results, previous reports have also shown a significant reduction in the number of stroke admissions [1-4, $8-12]$. However, the exact reason behind this decline is unclear. Nevertheless, there are some potential explanations in this regard. Many patients with minor symptoms of ischemic stroke and TIA likely become reluctant to seek medical emergency care or have a delayed hospital visit due to fear of COVID-19 infection in care facilities. This could increase the frequency of patients admitted with more severe conditions which is highly consistent with our findings. Significant changes in mortality rate, NIHSS, and GCS scores among ischemic stroke cases in our study indicated an increased proportion of patients admitted with severe symptoms in the COVID-19 pandemic period compared with the same period in 2019. Some previous investigations also had similar results regarding the changes in the severity of stroke $[1,10$, 11]. Similarly, a previous report from Brazil also showed a significant decrease in the rate of admission in mild cases of stroke [10]. Zini et al. in a study from Italy, also showed a significant increase in their percentage of moderate to severe stroke cases during the pandemic period [11]. Thus, these findings underscore the necessity for informing people particularly those at increased risk for ischemic stroke, of minor stroke symptoms and the importance of seeking medical care during the COVID-19 pandemic [13, 14].

Time from onset to arrival at the hospital was interestingly decreased in our ischemic stroke cases during the COVID-19 pandemic period. Accordingly, it is unlikely that delay in hospitalization has major effects on the severity of patients' symptoms. Therefore, the increase in the frequency of severe stroke cases is best explained by the reduced presentation of mild cases, given that the Poisson regression showed no significant correlation between the daily admissions of severe
Fig. 2 Weekly number of stroke admissions (all cases) with trends during pre-COVID-19 and COVID-19 periods

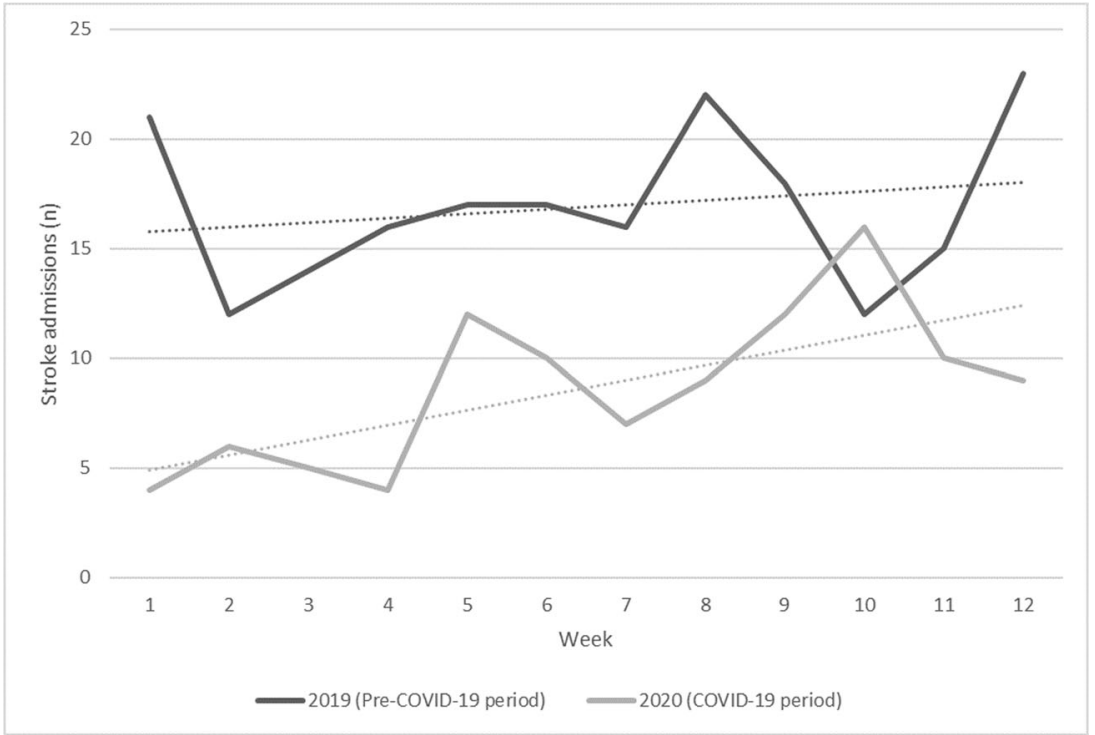


Table 3 Incidence rate ratios (IRRs) with 95\% confidence interval $(\mathrm{CI})$ calculated by Poisson regression (number of daily admissions as the response variable; month, day, and year as the independent variables)

\begin{tabular}{lllll}
\hline & Total IS cases & Mild IS cases & Severe IS cases & Hemorrhagic stroke cases \\
\hline Year IRR (95\% CI) & $0.51(0.40-0.64)$ & $0.34(0.25-0.46)$ & $1.34(0.86-2.08)$ & $0.55(0.26-1.15)$ \\
Month IRR (95\% CI) & $1.17(1.02-1.34)$ & $1.06(0.90-1.25)$ & $1.57(1.19-2.08)$ & $1.00(0.65-1.54)$ \\
Day IRR (95\% CI) & $0.98(0.97-0.99)$ & $0.98(0.97-0.99)$ & $0.99(0.96-1.01)$ & $1.00(0.97-1.04)$ \\
\hline
\end{tabular}

Year variable represents the COVID-19 and pre-COVID-19 periods stroke cases (NIHSS $\geq 9$ ) and the COVID-19 period, as well as a significantly reduced number of admissions in mild cases based on IRRs in our study. Patients with acute onset severe clinical deterioration are more likely to present to the emergency department than mild cases which could be the reason for the shorter time from onset to arrival. The unchanged time from onset to arrival, GCS scores, and the number of admissions between the two periods, for hemorrhagic cases (SAH and $\mathrm{ICH}$ ), further support this fact while taking into consideration the more severe presentation of these cases in comparison to ischemic stroke. Previous studies from Italy, Spain, and the USA with decentralized stroke networks, however, reported no change in time from onset to arrival $[1,11,12]$. As mentioned earlier, our stroke network follows a centralized model, and all eligible stroke cases are transferred to our center through the province-wide ambulance. One of the advantages of decentralized networks over centralized ones is the lower time from onset to arrival (prehospital delay) which is largely due to telestroke facilities. However, the shorter doorto-needle time (in-hospital delay) in centralized networks countervails it [15]. This finally results in a relatively similar time from onset to treatment in both networks. Road traffic reduction as an indirect effect of pandemic especially in urban areas also allows for a more rapid EMS response. Moreover, no substantial change in various components of our stroke network was found during the pandemic period. Hence, it is likely that due in part to the more pronounced effect of prehospital delays in centralized networks, we found a shorter time from onset to arrival during the pandemic period. Nevertheless, these findings should be interpreted cautiously owing to the effects of many confounding factors.

Furthermore, based on the results, it is unlikely that recent reorganizations in the healthcare system due to the COVID-19 pandemic have led to ineffective and delayed treatment with a resultant increase in in-hospital mortality. This is highly supported by the finding that time from arrival to thrombolysis and thrombectomy remained unchanged during the pandemic period. Moreover, the increase in the percentage of treatment procedures including thrombolysis, thrombectomy, and decompressive craniectomy was not significant during the pandemic period. Previous reports also demonstrated a similar door-to-needle time between the COVID-19 and preCOVID-19 periods [1, 11, 12]. Despite an unchanged doorto-needle time, Zini et al. showed a significant increase in door-to-scan time which was mainly due to COVID-19 screening measures according to authors [11]. However, Rudilosso et al. found no significant change in time from arrival to imaging between the two periods [12]. Although door-to-imaging time was not assessed in our study, we found a similar door-to-needle time between the pre-COVID-19 and COVID-19 periods which indicates that our in-hospital delay was not affected by the pandemic and screening measures.

We also examined the differences in clinical characteristics of ischemic stroke between the patients with positive and negative COVID-19 infection which was consistent with previous reports [16-20]. In contrast to the early reported cases [18] which included young patients without comorbid conditions, we found a higher mean age as well as high prevalence of preexisting comorbid conditions in COVID-19-positive stroke cases which is also supported by a systematic review of 26 studies involving 183 stroke patients with COVID-19 infection [19]. Similar to our study, an increase in the frequency of impaired level of consciousness as a presenting stroke symptom in COVID-19-positive cases compared with negative ones was reported previously. Our study also showed a significantly higher mortality rate and NIHSS scores in ischemic stroke cases with COVID-19 infection in comparison to non-infected ones. A previous large scale cohort study (the Global COVID-19 Stroke Registry) also reported findings similar to our study [16]. The time from COVID-19 symptoms to the onset of stroke in our study cohort was virtually similar to previous studies; however, $27.7 \%$ of stroke cases with COVID-19 infection were asymptomatic [19, 21]. In addition, we found a significantly higher frequency for cryptogenic stroke subtype among COVID-19-infected patients which is in line with previous reports $[11,20,22]$. Further, the rate of ischemic stroke cases presenting to emergency departments might be increased slightly during the pandemic period due to the higher burden of ischemic stroke with COVID-19 infection [20]. However, our study cohort included a small number of ischemic stroke cases with COVID-19 infection which was not the main objective of this study. Several prior reports have found an association between COVID-19 infection and some neurological symptoms such as peripheral nervous system involvement, encephalitis, myelitis, encephalopathy, and cerebrovascular disease $[21,23]$. Although the exact mechanism is still undetermined, there are several potential explanations including immune cell infiltration and inflammation due 
Table 4 Clinical and demographic characteristics of ischemic stroke cases with and without COVID-19 infection during the pandemic period

\begin{tabular}{|c|c|c|c|}
\hline & Positive COVID-19 infection & Negative COVID-19 infection & $P$ value \\
\hline Number of admissions & 15 & 80 & \\
\hline Age (mean, $\mathrm{SD}^{1}$ [years]) & $72.7(16.8)$ & $67.4(14.9)$ & 0.09 \\
\hline Sex $($ male $[n, \%])$ & $8(53.3 \%)$ & $42(52.5 \%)$ & 0.96 \\
\hline Time from onset to arrival at hospital (median $[\mathrm{IQR}]^{2}$, hours) & $6(3-18)$ & $6(4-15)$ & 0.98 \\
\hline \multicolumn{4}{|l|}{ Comorbidities $(n, \%)$} \\
\hline Hypertension & $7(46.7 \%)$ & $46(57.5 \%)$ & 0.43 \\
\hline Diabetes mellitus & $4(26.7 \%)$ & $26(32.5 \%)$ & 0.65 \\
\hline Coronary artery disease & $3(20.0 \%)$ & $23(28.8 \%)$ & 0.48 \\
\hline Heart failure & $1(6.7 \%)$ & $7(8.8 \%)$ & 0.79 \\
\hline Atrial fibrillation & $3(20.0 \%)$ & $15(18.8 \%)$ & 0.91 \\
\hline Renal disease & $1(6.7 \%)$ & $1(1.3 \%)$ & 0.18 \\
\hline Prior stroke & $3(20.0 \%)$ & $11(13.8 \%)$ & 0.53 \\
\hline Cancer & $0(0.0 \%)$ & $1(1.3 \%)$ & 0.66 \\
\hline Arterial territory (anterior/middle $[n, \%]$ ) & $8(53.3 \%)$ & $59(73.8 \%)$ & 0.11 \\
\hline \multicolumn{4}{|l|}{ Stroke subtypes $(n, \%)$} \\
\hline Large artery atherosclerosis & $3(20.0 \%)$ & $13(16.25 \%)$ & 0.71 \\
\hline Cardioembolic & $4(26.7 \%)$ & $18(22.5 \%)$ & 0.74 \\
\hline Small vessel disease & $0(0.0 \%)$ & $36(45.0 \%)$ & $0.001 *$ \\
\hline Other determined etiology & $0(0.0 \%)$ & $0(0.0 \%)$ & - \\
\hline Cryptogenic or undetermined & $8(53.3 \%)$ & $13(16.25 \%)$ & $0.004 *$ \\
\hline \multicolumn{4}{|l|}{ Symptoms $(n, \%)$} \\
\hline Impaired level of consciousness & $14(93.3 \%)$ & $34(42.5 \%)$ & $0.02 *$ \\
\hline Motor symptoms & $11(73.3 \%)$ & $59(73.8 \%)$ & 0.09 \\
\hline Sensory deficit & $0(0.0 \%)$ & $3(3.8 \%)$ & 0.44 \\
\hline Visual field loss & $0(0.0 \%)$ & $6(7.5 \%)$ & 0.27 \\
\hline Aphasia & $6(40.0 \%)$ & $17(21.3 \%)$ & 0.12 \\
\hline Dysarthria & $5(33.3 \%)$ & $33(41.3 \%)$ & 0.56 \\
\hline Ataxia & $0(0.0 \%)$ & $7(8.8 \%)$ & 0.23 \\
\hline \multicolumn{4}{|l|}{ Treatment interventions $(n, \%)$} \\
\hline Thrombolysis & $4(26.7 \%)$ & $14(17.5 \%)$ & 0.40 \\
\hline Thrombectomy & $2(13.3 \%)$ & $5(6.3 \%)$ & 0.33 \\
\hline Thrombolysis and thrombectomy & $1(6.7 \%)$ & $6(7.5 \%)$ & 0.91 \\
\hline \multicolumn{4}{|l|}{$\operatorname{NIHSS}^{3}(n, \%)$} \\
\hline Median (IQR) & $14(9.5-16.5)$ & $7.5(4-13)$ & $0.01 *$ \\
\hline $0-4$ & $2(13.3 \%)$ & $22(27.5 \%)$ & 0.34 \\
\hline $5-8$ & $2(13.3 \%)$ & $20(25.0 \%)$ & 0.50 \\
\hline $9-14$ & $5(33.3 \%)$ & $22(27.5 \%)$ & 0.75 \\
\hline$>14$ & $6(40.0 \%)$ & $14(17.5 \%)$ & 0.07 \\
\hline \multicolumn{4}{|l|}{$\operatorname{GCS}^{4}(n, \%)$} \\
\hline Mean (SD) & $10.27(2.74)$ & $13.18(2.60)$ & $<0.001^{*}$ \\
\hline $3-5$ & $1(6.7 \%)$ & $3(3.8 \%)$ & 0.50 \\
\hline $6-8$ & $2(13.3 \%)$ & $2(2.5 \%)$ & 0.11 \\
\hline $9-12$ & $9(60.0 \%)$ & $15(18.8 \%)$ & $0.002 *$ \\
\hline $13-15$ & $3(20.0 \%)$ & $56(70.0 \%)$ & $<0.001^{*}$ \\
\hline Decompressive craniectomy $(n, \%)$ & $0(0.0 \%)$ & $2(2.5 \%)$ & 1.00 \\
\hline In-hospital mortality $(n, \%)$ & $5(33.3 \%)$ & $9(11.3 \%)$ & $0.04 *$ \\
\hline
\end{tabular}

*indicates statistically significant $(P<0.05)$

${ }^{1}$ Standard deviation

${ }^{2}$ Interquartile range

${ }^{3}$ National Institute of Health Stroke Scale

${ }^{4}$ Glasgow coma scale

to viral CNS entry, virus-associated microangiopathy, endothelial dysfunction, and vasculitis [21-28]. Nevertheless, currently there is a lack of evidence regarding the pathophysiology and mechanism of neurological symptoms in COVID-19 infection, and there is a need for future high-quality studies to consider this. In this respect, a previous study has provided several recommendations for more accurate reporting of COVID-19-associated neurological manifestations [21].
The present study has some limitations. Firstly, due to the retrospective character of the study, it is difficult to determine the exact cause of the reduction in mild cases. The delay in presentation for mild cases of stroke is highly possible; however, it is hypothetical and more evidence from prospective studies is required. Secondly, this study was done in one stroke center. Despite providing new evidence regarding the changing pattern of admission in stroke, future nationwide 
cohort studies are highly needed to examine the differences in the epidemiology of stroke between the COVID-19 pandemic period and before.

\section{Conclusions}

This study revealed that there was a significant correlation between the COVID-19 pandemic period and a decreased number of stroke admissions. According to our findings, this reduction mostly involved mild cases (NIHSS $\leq 8$ ), and daily admission of more severe cases was not associated with the COVID-19 pandemic period. In addition to significantly increased severity in ischemic stroke cases due to a reduced number of admission in mild ones, a significantly decreased time from onset to arrival at the hospital was found. In conclusion, the present study results support the necessity of reorganization in the healthcare system especially stroke centers during the COVID-19 pandemic.

\section{Compliance with ethical standards}

Conflicts of interest The authors declare that they have no conflict of interest.

Ethical approval None

\section{References}

1. Siegler JE, Heslin ME, Thau L, et al (2020) Falling stroke rates during COVID-19 pandemic at a comprehensive stroke center: Cover title: Falling stroke rates during COVID-19. J Stroke Cerebrovasc Dis 29:104953. https://doi.org/10.1016/j. jstrokecerebrovasdis.2020.104953

2. Kansagra AP, Goyal MS, Hamilton S, Albers GW (2020) Collateral effect of covid-19 on stroke evaluation in the United States. N Engl J Med 383:400-401

3. Hoyer C, Ebert A, Huttner HB et al (2020) Acute stroke in times of the COVID-19 pandemic: a multicenter study. Stroke 51:2224 2227. https://doi.org/10.1161/STROKEAHA.120.030395

4. Mafham MM, Spata E, Goldacre R et al (2020) COVID-19 pandemic and admission rates for and management of acute coronary syndromes in England. Lancet 396:381-389. https://doi.org/10. 1016/S0140-6736(20)31356-8

5. Goyal M, Menon BK, Van Zwam WH et al (2016) Endovascular thrombectomy after large-vessel ischaemic stroke: a meta-analysis of individual patient data from five randomised trials. Lancet 387 : 1723-1731. https://doi.org/10.1016/S0140-6736(16)00163-X

6. Lees KR, Emberson J, Blackwell L et al (2016) Effects of alteplase for acute stroke on the distribution of functional outcomes: a pooled analysis of 9 trials. Stroke 47:2373-2379. https://doi.org/10.1161/ STROKEAHA.116.013644

7. Rothwell PM, Giles MF, Chandratheva A et al (2007) Effect of urgent treatment of transient ischaemic attack and minor stroke on early recurrent stroke (EXPRESS study): a prospective populationbased sequential comparison. Lancet 370:1432-1442. https://doi. org/10.1016/S0140-6736(07)61448-2
8. Uchino K, Kolikonda MK, Brown D et al (2020) Decline in stroke presentations during COVID-19 surge. Stroke:2544-2547. https:// doi.org/10.1161/STROKEAHA.120.030331

9. Hoyer C, Ebert A, Huttner HB et al (2020) Acute stroke in times of the COVID-19 pandemic. Stroke 51:2224-2227. https://doi.org/10. 1161/STROKEAHA.120.030395

10. Diegoli H, Magalhães PSC, Martins SCO et al (2020) Decrease in hospital admissions for transient ischemic attack, mild, and moderate stroke during the COVID-19 era. Stroke 51:2315-2321. https:// doi.org/10.1161/STROKEAHA.120.030481

11. Zini A, Romoli M, Gentile M, et al (2020) The stroke mothership model survived during COVID-19 era: an observational singlecenter study in Emilia-Romagna, Italy. Neurol Sci 1-5. https:// doi.org/10.1007/s10072-020-04754-2

12. Rudilosso S, Laredo C, Vera V et al (2020) Acute stroke care is at risk in the era of COVID-19: 3xperience at a comprehensive stroke center in Barcelona. Stroke 51:1991-1995. https://doi.org/10.1161/ STROKEAHA.120.030329

13. Lyden P (2020) Temporary emergency guidance to US stroke centers during the coronavirus disease 2019 (COVID-19) pandemic: on behalf of the American Heart Association/American Stroke Association Stroke Council Leadership. Stroke 51:1910-1912

14. Zhao J, Rudd A, Liu R (2020) Challenges and potential solutions of stroke care during the coronavirus disease 2019 (COVID-19) outbreak. Stroke 51:1356-1357

15. Hubert GJ, Meretoja A, Audebert HJ et al (2016) Stroke thrombolysis in a centralized and a decentralized system (Helsinki and Telemedical Project for Integrative Stroke Care Network). Stroke 47:2999-3004. https://doi.org/10.1161/STROKEAHA.116. 014258

16. Ntaios G, Michel P, Georgiopoulos G, et al (2020) Characteristics and outcomes in patients with COVID-19 and acute ischemic stroke: the global COVID-19 stroke registry. Stroke 51:e254 e258. https://doi.org/10.1161/STROKEAHA.120.031208

17. Majidi S, Fifi JT, Ladner TR et al (2020) Emergent large vessel occlusion stroke during New York City's COVID-19 outbreak. Stroke 51. https://doi.org/10.1161/strokeaha.120.030397

18. Yaghi S, Ishida K, Torres J, et al (2020) SARS-CoV-2 and stroke in a New York healthcare system. Stroke 2002-2011. https://doi.org/ 10.1161/STROKEAHA.120.030335

19. Yamakawa M, Kuno T, Mikami T et al (2020) Clinical characteristics of stroke with COVID-19: a systematic review and metaanalysis. J Stroke Cerebrovasc Dis 29:105288. https://doi.org/10. 1016/j.jstrokecerebrovasdis.2020.105288

20. Katsanos AH, Palaiodimou L, Zand R et al (2020) The impact of SARS-CoV -2 on stroke epidemiology and care: a meta-analysis. Ann Neurol. https://doi.org/10.1002/ana.25967

21. Romoli M, Jelcic I, Bernard-Valnet R et al (2020) A systematic review of neurological manifestations of SARS-CoV-2 infection: the devil is hidden in the details. Eur J Neurol 27:1712-1726. https://doi.org/10.1111/ene.14382

22. Yan R, Zhang Y, Li Y et al (2020) Structural basis for the recognition of SARS-CoV-2 by full-length human ACE2. Science 367: 1444-1448. https://doi.org/10.1126/science.abb2762

23. Ellul MA, Benjamin L, Singh B et al (2020) Neurological associations of COVID-19. Lancet Neurol 19:767-783

24. Brisse E, Wouters CH, Andrei G, Matthys P (2017) How viruses contribute to the pathogenesis of hemophagocytic lymphohistiocytosis. Front Immunol 8. https://doi.org/10.3389/ fimmu.2017.01102

25. da Silva RL (2011) Viral-associated thrombotic microangiopathies. Hematol Oncol Stem Cell Ther 4:51-59

26. Varga Z, Flammer AJ, Steiger P et al (2020) Endothelial cell infection and endotheliitis in COVID-19. Lancet 395:1417-1418

27. Chu H, Chan JF-W, Yuen TT-T et al (2020) Comparative tropism, replication kinetics, and cell damage profiling of SARS-CoV-2 and 
SARS-CoV with implications for clinical manifestations, transmissibility, and laboratory studies of COVID-19: an observational study. Lancet Microbe 1:e14-e23. https://oi.org/10.1016/s26665247(20)30004-5

28. Hamming I, Timens W, Bulthuis MLC et al (2004) Tissue distribution of ACE2 protein, the functional receptor for SARS coronavirus. A first step in understanding SARS pathogenesis. J Pathol 203:631-637. https://doi.org/10.1002/path.1570

Publisher's note Springer Nature remains neutral with regard to jurisdictional claims in published maps and institutional affiliations. 